\title{
Comparison Study of a-SiGe Solar Cells and Materials Deposited Using Different Hydrogen Dilution
}

\author{
H. Povolny, P. Agarwal, S. Han, and X. Deng \\ Department of Physics and Astronomy, University of Toledo, Toledo, OH 43606
}

\begin{abstract}
A-SiGe n-i-p solar cells with i-layer deposited via plasma enhanced chemical vapor deposition (PECVD) with a germane to disilane ratio of 0.72 and hydrogen dilution $\mathrm{R}=\left(\mathrm{H}_{2}\right.$ flow $) /\left(\mathrm{GeH}_{4}+\mathrm{Si}_{2} \mathrm{H}_{6}\right.$ flow $)$ values of $1.7,10,30,50,120,180$ and 240 were deposited on stainless steel substrates. This germane to disilane ratio is what we typically use for the i-layer in the bottom cell of our standard triple-junction solar cells. Solar cell current-voltage curves (J-V) and quantum efficiency $(\mathrm{QE})$ were measured for these devices. Light soaking tests were performed for these devices under 1 sun light intensity at $50^{\circ} \mathrm{C}$. While device with $\mathrm{R}=30$ showed the highest initial efficiency, the device with $\mathrm{R}=120$ exhibit higher stabilized efficiency after 1000 hours of light soaking.

Single-layer a-SiGe films ( $\sim 500 \mathrm{~nm}$ thick) were deposited under the same conditions as the i-layer of these devices on a variety of substrates including 7059 glass, crystalline silicon, and stainless steel for visible-IR transmission spectroscopy, FTIR, and hydrogen effusion studies. It is interesting to note 1) the $\mathrm{H}$ content in the film decreased with increasing $\mathrm{R}$ based on both the $\mathrm{IR}$ and $\mathrm{H}$ effusion measurements, and 2 ) while the $\mathrm{H}$ content changes significantly with different $\mathrm{R}$, the change in $\mathrm{E}_{\mathrm{g}}$ is relatively small. This is most likely due to a change in Ge content in the film for different $\mathrm{R}$.
\end{abstract}

\section{INTRODUCTION}

Although the correlation between amorphous silicon based solar cell device performance and a variety of intrinsic layer (i-layer) materials properties have been studied broadly [1-3], a reliable correlation between i-layer properties and device performance is not yet well established. This is partially due to the different ways used for depositing these materials. H dilution has been used extensively to improve a-Si based solar cell materials and device quality [4-6]. However, the exact role of $\mathrm{H}$ dilution to the growth of a-Si alloys, in particular narrow bandgap a-SiGe materials, and its impact on the solar cell device performance and single-layer film properties are not yet fully understood and deserve further study. In this paper, we describe our recent studies of a-SiGe n-i-p solar cells and films deposited under a wide range of $\mathrm{H}$ dilution. We were particularly interested in the effect of the different hydrogen dilution on long-term stability of these solar cells.

\section{EXPERIMENT}

A-SiGe films, approximately $0.5 \mu \mathrm{m}$ thick, were deposited on 7059 glass, c-Si substrates and stainless steel (SS) using a hydrogen dilution ratio R of 1.7, 10, 30, 50, 120, 180 and 240 using PECVD. Optical measurements were performed for samples on 7059 glass substrates to obtain sample thickness, refractive index and the bandgap. FTIR absorption measurements were taken for samples on c-Si substrates to obtain information on the $\mathrm{H}$ content and bonding. $\mathrm{H}$ 
effusion measurements were performed on samples deposited on SS substrates to obtain the total H content.

Single junction n-i-p solar cells using these a-SiGe materials as the i-layers were deposited on SS substrates without the use of a back-reflector. I-V measurements were taken under a Xelamp solar simulator under AM1.5 spectrum. Quantum efficiency (QE) measurements were taken between $420 \mathrm{~nm}$ and $900 \mathrm{~nm}$. Light soaking was performed under one-sun light intensity using a metal halide lamp. The intensity is maintained at a level so that a reference Si solar cell generates the same current as it does under a Xe-lamp simulator.

\section{RESULTS}

\section{Single-layer a-SiGe films}

Table I shows the deposition conditions for these a-SiGe films including the flows of $\mathrm{GeH}_{4}$, $\mathrm{Si}_{2} \mathrm{H}_{6}$ and $\mathrm{H}_{2}$, the substrate temperature, radio frequency power, chamber pressure, and deposition time. The deposition times of our single layer films were adjusted so that the samples would have approximately the same thickness. It is worth noting that Sample GD422 is made using the dilution that is currently used for the bottom cell i-layer and, therefore, serves as a reference point for this set of samples.

Transmission spectra of the single layers were measured using dual beam visible/near-IR spectroscopy. No substrate was used in the reference beam during the measurement. There were clear interference fringes within the measured range allowing us to calculate the refractive index $n$ in the weak absorption region and the thickness $d$ for the samples [7]. A linear fit to the refractive index $n$ plotted versus $1 / \lambda^{2}$ allows us to extrapolate the $n$ value in the strong absorption region $(\lambda<800 \mathrm{~nm})$. The $n$ values estimated from this extrapolation were then used together with the transmission values in the strong absorption region to obtain absorption coefficient $\alpha$. A linear fitting of the Tauc plot, $(\alpha h v)^{1 / 2}$ vs. hv, gives the material's bandgap $\mathrm{E}_{\mathrm{g}}$.

Using the above procedure, $n, d$, and $E_{g}$ for all of the samples with different $\mathrm{R}$ were measured and summarized in Table II. The deposition rates were obtained from the thicknesses and deposition times. It is found that the deposition rate is reduced by more than one order of magnitude when $\mathrm{R}$ is increased from 1.7 to 240 .

To find out $\mathrm{H}$ content and the bonding configuration, infrared absorption for these films

Table I. Deposition conditions for the single i-layer including process gas flow rates, temperature, radio-frequency power, chamber pressure, and deposition time.

\begin{tabular}{|c|c|c|c|c|c|c|c|c|}
\hline Sample & $\mathrm{R}$ & $\mathrm{GeH}_{4}$ & $\mathrm{Si}_{2} \mathrm{H}_{6}$ & $\mathrm{H}_{2}$ & Temp & Power & Pressure & Time \\
\hline No. & & $(\mathrm{sccm})$ & $(\mathrm{sccm})$ & $(\mathrm{sccm})$ & $(\mathrm{C})$ & $(\mathrm{W})$ & $($ Torr $)$ & $(\mathrm{min})$ \\
\hline GD419 & 1.7 & 0.72 & 1 & 2.9 & 400 & 3 & 0.6 & 30 \\
\hline GD420 & 10 & 0.72 & 1 & 17.2 & 400 & 3 & 0.6 & 60 \\
\hline GD422 & 30 & 0.72 & 1 & 51.6 & 400 & 3 & 0.6 & 120 \\
\hline GD423 & 50 & 0.72 & 1 & 86.0 & 400 & 3 & 0.6 & 180 \\
\hline GD424 & 120 & 0.72 & 1 & 202 & 400 & 3 & 0.6 & 240 \\
\hline GD449 & 180 & 0.468 & 0.65 & 202 & 400 & 3 & 0.6 & 360 \\
\hline GD450 & 240 & 0.35 & 0.49 & 201 & 400 & 3 & 0.6 & 480 \\
\hline
\end{tabular}


Table II. Results of measurements on single i-layer films including thickness of film, index of refraction, bandgap, and hydrogen content.

\begin{tabular}{|c|c|c|c|c|c|c|c|}
\hline Sample & $\mathrm{R}$ & Thickness & Dep.rate & $\mathrm{n} @ 600 \mathrm{~nm}$ & $\mathrm{E}_{\mathrm{g}}$ & {$[\mathrm{H}]$ from IR } & H effusion \\
\hline No. & & $(\mathrm{nm})$ & $(\mathrm{nm} / \mathrm{sec})$ & & $(\mathrm{eV})$ & $($ at.\%) & relative \\
\hline GD419 & 1.7 & 529 & 0.294 & 4.42 & 1.515 & 15.4 & 13.8 \\
\hline GD420 & 10 & 531 & 0.148 & 4.45 & 1.489 & 12.4 & 9.7 \\
\hline GD422 & 30 & 556 & 0.0772 & 4.36 & 1.479 & 10.9 & 13.2 \\
\hline GD423 & 50 & 608 & 0.0563 & 4.29 & 1.482 & 8.6 & 7.9 \\
\hline GD424 & 120 & 410 & 0.0285 & 4.48 & 1.516 & 11.6 & 7.6 \\
\hline GD449 & 180 & 492 & 0.0227 & 4.47 & 1.501 & 5.1 & -- \\
\hline GD450 & 240 & 511 & 0.0177 & 4.53 & 1.482 & 4.7 & -- \\
\hline
\end{tabular}

were measured using an FTIR spectroscope [8]. There were absorption peaks at 640 and 2000 $\mathrm{cm}^{-1}$, corresponding to the waging and stretching modes of Si-H bonding. Ge-H peaks, $560 \mathrm{~cm}^{-1}$ for waging mode and $1975 \mathrm{~cm}^{-1}$ for the stretching mode, are buried in the Si-H peaks. From the $640 \mathrm{~cm}^{-1}$ (plus $560 \mathrm{~cm}^{-1}$ ) peak we calculated the $\mathrm{H}$ content. We find that the $\mathrm{H}$ content generally decreases with increasing R. Sample GD419 (with R=1.7) has the largest amount of H. While there is a pronounced peak near $2100 \mathrm{~cm}^{-1}$ for the low R sample (GD 419), the stretching mode absorption for the high $\mathrm{R}$ sample $(\mathrm{R}=120)$ shows only $2000 \mathrm{~cm}^{-1}$ absorption indicating mostly monohydride bonding.

Figure 1 shows the $\mathrm{H}$ effusion spectra of these a-SiGe films. The partial pressure of $\mathrm{H}$ is measured during pumping so the $\mathrm{H}$ content is proportional to the integrated area under the curves. A relative value from the integrated area is shown in Table II. Again, we see that the $\mathrm{H}$ content generally decreases with increasing $\mathrm{R}$.

It is interesting to note that while $\mathrm{H}$ content is decreased significantly, for example from 15.4 at. $\%$ for $\mathrm{R}=1.7$ to 4.7 at. $\%$ for $\mathrm{R}=240$ as determined by FTIR, the bandgap remains approximately the same. This is surprising since such a sizable drop in $\mathrm{H}$ content would lead to a large drop in $\mathrm{E}_{\mathrm{g}}$. The lack of sizable change in $\mathrm{E}_{\mathrm{g}}$ suggests that the Ge contents in the high $\mathrm{R}$ samples are likely lower. Further experiments will be performed to verify this.

\section{a-SiGe n-i-p Devices}

The a-SiGe films with different $\mathrm{R}$ were used as i-layer for $\mathrm{n}-\mathrm{i}-\mathrm{p}$ solar cells. The device structure was: SS/a-Si n ${ }^{+} / \mathrm{n}$-i buffer/a-SiGe absorber layer/i-p buffer/ $\mu \mathrm{c}-\mathrm{Si} \mathrm{p}^{+} / \mathrm{ITO}$. The n-i and i$\mathrm{p}$ buffer layers consisted of a-Si buffer layers next to the doped layer and a-SiGe interface layers with a graded bandgap to reduce bandgap discontinuity. The a-SiGe absorber layer had a graded bandgap such that the bandgap is narrower near the p-side [9]. Such a graded bandgap is used in the routine fabrication of bottom cells for high efficiency triple-junction solar cells. No backreflector was used for this study since the comparative study would be expected to yield the same results with or without a back-reflector.

For all of these devices, only the i-layer conditions were varied. All other deposition conditions were kept unchanged. In order to fabricate solar cells with i-layer of approximately the same thickness, the i-layer deposition time was adjusted according to the thickness measurement of the single-layer sample. The thickness of the i-layer was measured using a capacitance technique. This thickness includes the thickness of both the a-SiGe absorber layer 


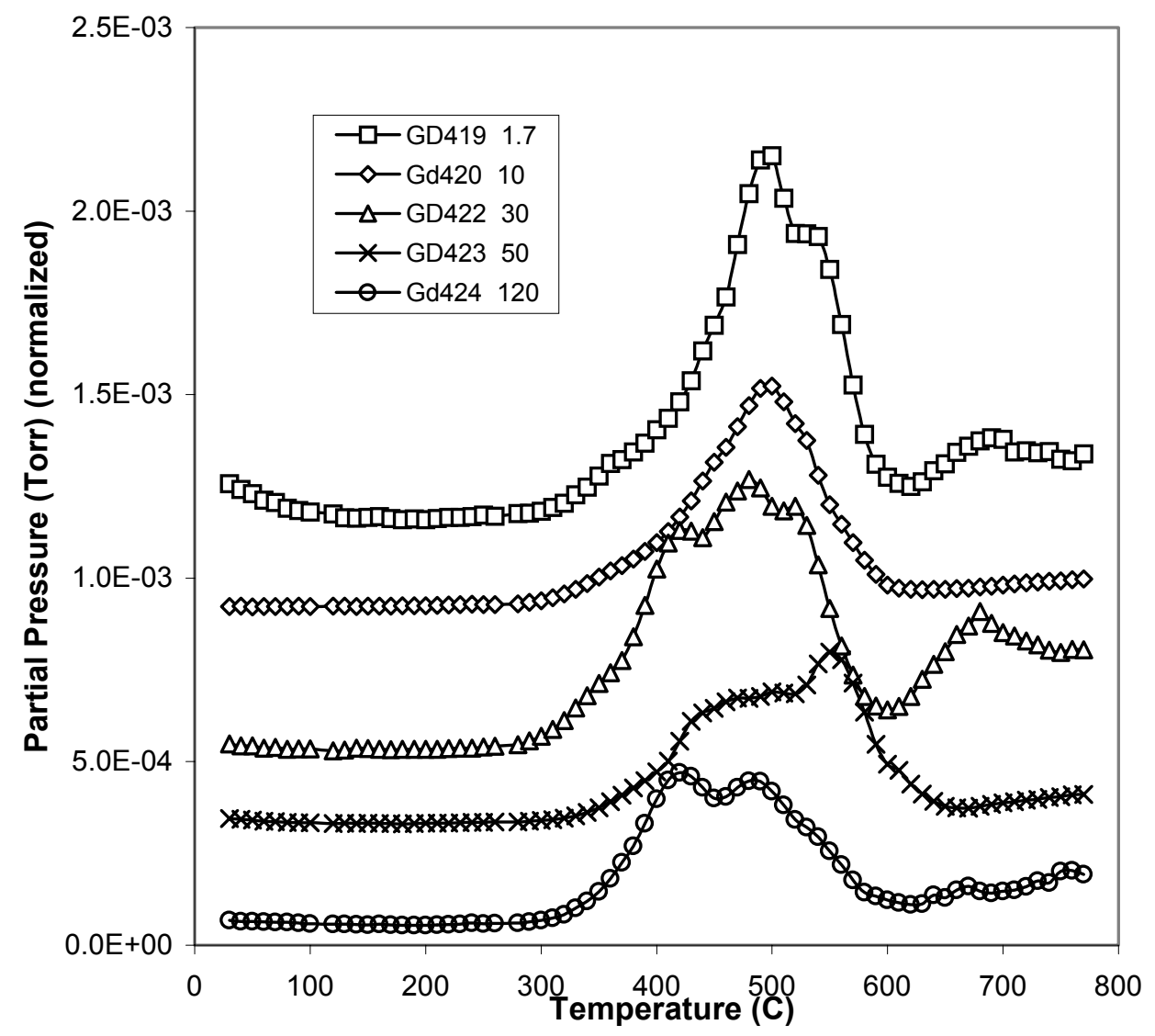

Figure 1. Results of $\mathrm{H}$ effusion experiments performed under differential pumping. Total $\mathrm{H}$ content is the area under the curve.

and the buffer layers. Table III shows the solar cell performance of a series of a-SiGe solar cells deposited using different $\mathrm{H}$ dilution, ranging from 1.7 to 240 . The $\mathrm{V}_{\mathrm{oc}}$ increases from $0.64 \mathrm{~V}$ to $0.67 \mathrm{~V}$ with $\mathrm{R}$ up to 120 , before it starts to decrease down to $0.64 \mathrm{~V}$ when $\mathrm{R}$ is further increased to 240 . This may suggest that small amount of microcrystalline phase is formed at $\mathrm{R}=240$. While it is surprising to find that at $\mathrm{R}=240$ the film is still somewhat amorphous, it is realized that the a-SiGe films were deposited in a chamber that has been used to deposit many runs of narrow bandgap a-SiGe films. There could be contribution of unnegligible amount of Ge containing species in the plasma from the chamber walls in addition to those from $\mathrm{GeH}_{4}$ gas. However, all deposition reactors, including a-Si PV production machines, will have chamber walls coated with the materials to be deposited. The $\mathrm{J}_{\mathrm{sc}}$ is roughly unchanged within the experimental error, which is approximately $5-10 \%$. The $\mathrm{J}_{\mathrm{sc}}$ is relatively low since no back-reflector is used for this study. The FF is highest for $\mathrm{R}=30$, a moderate dilution, which is the condition that we used for the fabrication of the bottom cell of our 11\% triple-junction solar cell [10]. Device GD425 ( $\mathrm{R}=30)$ is the same as our standard bottom cells and is used as a reference for this dilution study.

$\mathrm{J}-\mathrm{V}$ curves of the cells were measured under a Xenon lamp solar simulator with AM1.5 light. The quantum efficiency $(\mathrm{QE})$ for this set of devices with different $\mathrm{R}$ was also measured. For a-SiGe solar cells deposited on bare SS, the red response was high. In Table III, we 
Table III. Performance of n-i-p devices made with different $\mathrm{H}$ dilution R.

\begin{tabular}{|c|c|c|c|c|c|c|c|c|c|c|c|}
\hline Device & $\begin{array}{c}\mathrm{H} \\
\text { dilution }\end{array}$ & Time & Thickness & $\mathrm{V}_{\mathrm{oc}}$ & $\mathrm{J}_{\mathrm{sc}}$ & $\mathrm{FF}$ & Eff & QE & QE & QE $_{\max }$ & $\begin{array}{c}\text { Light } \\
\text { Degrad. }\end{array}$ \\
\hline No. & $\mathrm{R}$ & $(\mathrm{min})$ & $(\mathrm{nm})$ & $(\mathrm{V})$ & $\left(\mathrm{mA} / \mathrm{cm}^{2}\right)$ & $(\%)$ & $(\%)$ & at $400 \mathrm{~nm}$ & at $700 \mathrm{~nm}$ & & $(\%)$ \\
\hline GD429 & 1.7 & 9.5 & 189 & 0.638 & 16.2 & 53.8 & 5.54 & 0.57 & 0.37 & 0.81 & $>21.1$ \\
\hline GD428 & 10 & 19 & 190 & 0.640 & 17.7 & 53.1 & 6.02 & 0.58 & 0.42 & 0.83 & 20.9 \\
\hline GD425 & 30 & 36 & 189 & 0.656 & 17.4 & 59.1 & 6.76 & 0.59 & 0.43 & 0.83 & 21.6 \\
\hline Gd426 & 50 & 50 & 175 & 0.662 & 17.2 & 55.0 & 6.25 & 0.58 & 0.32 & 0.82 & 14.2 \\
\hline GD427 & 120 & 98 & 190 & 0.669 & 17.0 & 54.8 & 6.25 & 0.60 & 0.39 & 0.84 & 11.4 \\
\hline GD451 & 180 & 123 & 176 & 0.656 & 18.1 & 56.0 & 6.65 & 0.64 & 0.27 & 0.80 & -- \\
\hline GD452 & 240 & 158 & 168 & 0.636 & 18.5 & 55.8 & 6.55 & 0.63 & 0.29 & 0.79 & -- \\
\hline
\end{tabular}

summarize some of the QE data including the maximum of QE, the QE values at $400 \mathrm{~nm}$ and $700 \mathrm{~nm}$. The results are largely consistent.

\section{Light Soaking Stability Test}

Light soaking tests for these a-SiGe samples were performed to understand the effect of $\mathrm{R}$ on the device stability. The light soaking was done under a metal halide lamp with one-sun light intensity. This lamp is relatively blue rich and has somewhat less red light compared with AM1.5 global spectrum. Our samples are light soaked under one-sun light intensity. Since an a-SiGe bottom cell typically sees only one third of the sun light inside a triple-junction solar cell, this one-sun light intensity is approximately three times more intense than that in the standard testing conditions. However, the relative trend among these samples would likely remain the same. The last column of Table III shows the degradation under 1 sun light soaking at $50^{\circ} \mathrm{C}$ for 1000 hours. The degradation for sample GD429 at 1000 hours of soaking is unavailable since all of the test cells were shorted. The 300 -hour soaking results shows a degradation of $21.1 \%$, which is expected to be further increased at 1000 hours. It is found that the degradation is reduced with higher $\mathrm{R}$, down from $20.9 \%$ for $\mathrm{R}=10$ to $11.4 \%$ for $\mathrm{R}=120$. Degradation of Samples GD451 and GD452 are not included here since they were fabricated later. From Table III, one concludes that before light soaking, $\mathrm{R}=30$ (GD425) shows the highest $\mathrm{P}_{\max }$ while after light soaking, $\mathrm{R}=120$ (GD427) shows the highest $P_{\max }$.

\section{CONCLUSION}

We have deposited a series single-layer films and n-i-p solar-cell devices using narrow bandgap $(\sim 1.5 \mathrm{eV})$ a-SiGe materials deposited via PECVD using different $\mathrm{H}$ dilution ranging from $\mathrm{R}=1.7$ to 240 . Samples with higher $\mathrm{R}$ value showed lower amounts of $\mathrm{H}$ incorporated into the film though the bandgap remained approximately the same. This suggests that the Ge content in films deposited with higher $\mathrm{R}$ is likely lower. Measurements of Ge content in these films will be performed to verify this. The results from the devices and films deposited using different $\mathrm{H}$ dilution suggest that a high $\mathrm{H}$ dilution up to 120 could be used to deposit a-SiGe films with improved light stability. 


\section{ACKNOWLEDGMENTS}

This work is supported by NREL under the Thin Film Partnership Program (No. ZAF-817619-14). The authors are grateful for the help from Dr. A. D. Compaan and our colleagues at ECD and United Solar. The authors are also grateful to Dr. S. C. Agarwal at the Indian Institute of Technology, Kanpur for the use of his H-effusion system.

\section{REFERENCES}

1. L. Yang, L. Chen, and A. Catalano, MRS Proc. 219, 259 (1991).

2. J. Yang, X. Xu and S. Guha, MRS Proc. 336, 687 (1994).

3. M. Shima, M. Isomura, E. Maruyama, S. Okamoto, H. Haku, K. Wakisaka, S. Kiyama, and S. Tsuda, MRS Proc. 507, 145 (1998).

4. S. Guha, K.L. Narasimhan, and S.M. Pietruszko, J. Appl. Phys. 52, 859 (1981).

5. K. Tanaka and A. Matsuda, MRS Proc. 70, 245 (1986).

6. J. Yang and S. Guha, MRS Proc. 557, 239 (1999).

7. R. Swanepoel, J. Phys. E: Sci. Instrum. 16, pp. 1214-1222 (1983).

8. A. D. Compaan and X. Deng, University of Toledo $19992^{\text {nd }}$ Quarterly Report submitted to NREL under the Thin Film Partnership Program (1999).

9. J. Yang, R. Ross, T. Glatfelter, R. Mohr, and S. Guha, MRS Proc. 149, 435 (1989).

10. X. Deng, X. B. Liao, S. Han, H. Povolny, P. Agarwal, Solar Energy Mat. \& Solar Cells, 2000 . 